Lepr Rev (1998) 69, 2-5

\title{
Editorial
}

\section{IS THERE A MICROBIOLOGICAL RATIONALE FOR SINGLE-DOSE TREATMENT OF LEPROSY?}

Therapy of any infectious disease is based on some general principles: (i) administration of appropriate $\operatorname{drug}(\mathrm{s})$ to which the organism is susceptible; (ii) $\operatorname{drug}(\mathrm{s})$ should be able to reach the site of action and stay in the system long enough; (iii) therapy may need to be repeated to reduce the viable load below detectable limits, i.e. culture negativity. In various bacterial diseases, treatment is continued beyond the point of achieving sterility so that relapses are reduced to acceptably low limits. These principles have been followed for the management of tuberculosis when treatment is given for 3-4 times the duration required for achieving culture negativity. The principle is to eliminate both metabolically active and dormant persisters to a level where host immunity disposes of residual organisms. These aspects have been considered when planning various multidrug therapy (MDT) regimens for leprosy during the last 15 years. ${ }^{1,2}$ However, as Mycobacterium leprae is not cultivable in-vitro theoretical considerations have been based on the results of multiplication and killing/inhibition in the mouse footpad model. The treatment of leprosy has progressed through many phases. Drugs like rifampicin, dapsone, and clofazimine were initially selected on the basis of mouse footpad experiments. Based on the calculations of the proportion of $M$. leprae killed after one or more doses of rifampicin, regimens were designed to kill an estimated viable load and to prevent the emergence of drug resistance. These regimens (popularly known as WHO regimens) have been used all over the world and have led to a major decline in the prevalence of the disease. ${ }^{3,4}$ But after reduction of well-established (tuberculoid and lepromatous) cases, the spectrum of the disease has shifted to fresh evolving cases and single-lesion cases. The encouraging experience with MDT and availability of newer potent compounds has given confidence to try ever shorter regimens. ${ }^{3,5}$ This thinking has culminated in the development of a single-dose regimen comprising of rifampicin, ofloxacin and minocycline (ROM) for the treatment of singlelesion cases. This regimen has now been recommended for implementation in treatment programmes, so that such cases can be declared 'cured' before the deadline of 2000 AD. This regimen is really 'revolutionary' and moves from a era of life-long therapy to one-day treatment. This has also stirred up much needed debate which the earlier WHO regimens had escaped. Debate on the rational of the new ROM regimen needs to consider the experience and lessons from the earlier WHO regimen(s) as the justification for ultrashort regimens is based on the overall 'success' of the older regimens. 


\section{Experience with current/old WHO regimens}

Published results show that WHO regimens were generally more effective than dapsone monotherapy in terms of faster clinical response, faster reduction of viability and lower rates of late complications like relapses. ${ }^{3,4}$ The initial recommendations of treatment for a minimum of 6 months for paucibacillary (PB) cases and 2 years for multibacillary (MB) leprosy worked in the majority of cases. However, several difficulties and limitations in both the management of PB and MB leprosy with these regimens have also become apparent. ${ }^{6}$ There are problems of residual persisting activity and late reactions/relapses in paucibacillary cases treated with 6-months fixed-duration regimen. ${ }^{7}$ The problem of persisting viable bacilli 'persisters' in a significant proportion of initially highly-bacillated MB cases was also observed by several investigators. ${ }^{8-11}$ These results may be interpreted in various ways. While rapid decrease in viable populations as seen in the mouse footpad and other viability parameters was a heartening development, it was apparent that killing efficiency is not always predicted by the mouse footpad. If the calculations of $99.9 \%$ killing with a single dose of rifampicin were also reproducible in man, then there would have been no problem. It was observed that there was a rapid decrease in viable populations initially but little effect on dormant or nonmultiplying bacillary populations. Viable bacteria could be detected or grown in about $>10 \%$ of these cases after 3-24 months of treatment. ${ }^{8-11}$ As the potent bactericidal drug rifampicin only stays in the system for a couple of hours, this can only eliminate bacteria multiplying on that day. These limitations occur inspite of one (dapsone) or two drugs (dapsone and clofazimine) being administered continuously. The presence of these persisters and the possible consequences have not been addressed. It has been assumed that the host response would eliminate residual viable bacteria. It was also postulated that after the initial doses there were no additional benefits of MDT as a continuous decline in bacteriological indices is seen. ${ }^{12}$ It is unlikely that the effect of 3 months, 12 months or 24 months treatment is the same. Inability to discriminate between the outcome of different treatment times is probably due to inadequate follow-up and the limitation of testing methods. The situation in paucibacillary leprosy is not severe, the premature termination of treatment leads to persistence of activity, late complications like late reactions/relapses in a section of cases which could have been reduced by appropriate modifications. ${ }^{7}$ But in multibacillary cases, the consequences have been worse. In trials with excellent follow-up (e.g. Marchoux trial) relapses have occurred almost in the same proportion as the persisters were seen in various studies and most of these relapses occurred 6-9 years later. ${ }^{13}$ This experience shows that bacteriological aspects have to be considered and it is wrong to draw overoptimistic conclusions especially in cases with demonstrable proof of viable bacilli. Leaving behind live organisms may be a risky idea in leprosy and should be kept in mind while planning regimes in present or future.

\section{Management of single-lesion cases with single-dose treatment}

Rifampicin, ofloxacin and minocycline are well-established drugs which have shown reasonably high bactericidal activity against $M$. leprae. Even though there are no indepth investigations, it has been generally assumed that single-lesion cases are paucibacillary cases with a good immunity and low bacterial load. ${ }^{14,15}$ As discussed above, experience with current MDT shows that these calculations and assumptions do apply regardless. Despite the 
presence or one or a few skin lesions, M. leprae may be multiplying in internal organs and the actual load may be much higher.

Assuming that a particular case has $10-100$ million organisms and if $0 \cdot 1-1 \cdot 0 \%$ are left unkilled, this leaves a million live bacilli. The drugs only act on metabolically-active organisms and the half lives of these drugs vary from 3 to 5 hours for rifampicin, to 7 to 8 hours for ofloxacin, and 12-18 hours for minocycline. Therefore, these drugs will only eliminate organisms which are metabolically active during that period when these drugs are in the body. These compounds may act synergistically to achieve better killing kinetics. They will also prevent the development of drug-resistant organisms to these compounds. However the bacterial populations which are not in the growth phase or are dormant will not be affected by this drug combination. The result will be possibly the same even if $2-3$ more compounds are added up.

The results of the multicentre trial show that this regimen is well tolerated and with few immediate problems. However, in a short follow-up of 18 months, the clinical response has been slower than with the current WHO regimen and more than $50 \%$ of cases were still active. ${ }^{15}$ If one compares experience with the previous studies, the response of single-lesion cases has been much better to WHO regimen or slightly modified regimens. ${ }^{16-18}$ Nearly $95-$ $100 \%$ inactivity was achieved in those trials by one year. ${ }^{16-18}$ In the present case even the response to current WHO regimen has not been that good. One interpretation could be that we are dealing with a different situation. In the earlier era, most of the single-lesion cases were established tuberculoid cases with some immunity to limit disease. However, the new singlelesion cases may be evolving early cases - and they may progress into lepromatous or tuberculoid types. The relatively slower response to the WHO regimen itself may support this hypothesis. Some of the single-lesion cases may self-heal, ROM may accelerate healing in some by killing some metabolically active $M$. leprae but in others there may be no antibacterial effect. Leprosy lesions are not a synchronous culture of bacteria which can be instantaneously killed. It may be inappropriate to consider all these cases as low-bacillated 'paucibacillary' with their $M$. leprae actively multiplying and easily killed when drugs are administered. Synchronous cultures of cultivable mycobacteria are extremely difficult to generate and sustain even in the artificial test tube conditions. The experience with MDT regimens in $\mathrm{MB}$ as well as $\mathrm{PB}$ cases shows clearly that we are not dealing with synchronous actively multiplying organisms. Whether $M$. leprae multiplies after 10-12 days or 1-2 days (as per calculations of Dr Hastings) may be debatable but it is certain that all M. leprae do not multiply on the same day. The number of required pulses cannot be predicted with certainty and can only be decided by well-conducted trials. It is rather strange to base therapy on the killing observed in the mouse footpad but conveniently choose to ignore the presence of viable $M$. leprae demonstrated by the same technique. The ROM regimen has a poor microbiological rational which cannot be theoretically defended. This has been further substantiated by a relatively slower response as seen in the trial.

In the changing era of leprosy elimination when a major chunk of cases has already been eliminated, at least from the registers, it is difficult to understand the logic of designing and rushing with a regimen with poor theoretical chance of achieving reasonable antibacterial effect. As the initial response has been below the expected levels, large scale introduction of this regimen in the field may have serious consequences. Long term follow-up (up to 10 years as some of them could be potential MB types) and indepth debate about the likely consequences should be carried out. While in doubt, the benefit should rest with the patient and not with theoretical optimism. 
Central JALMA Institute for Leprosy (ICMR)

V. M. KATOCH

Tajganj

Agra-282001, India

\section{References}

${ }^{1}$ Chemotherapy of Leprosy for control programmes. Report of WHO study group, Geneva, World Health Organisation, 1982. Tech Rep Ser 675.

2 WHO Expert Committee on Leprosy. Sixth Report, Geneva, World Health Organisation, 1988. Tech Rep Ser 768.

${ }^{3}$ Grosset JH. Progress in the chemotherapy of leprosy. Int J Lepr, 1994; 62: 268-77.

${ }^{4}$ Noordeen SK. Elimination of leprosy as a public health problem. Int J Lept, 1994; 62: 278-83.

5 APEL, WHO. Shortening of treatment of multibacillary leprosy. Indian J Lepr, 1997; 69: 267-70.

${ }^{6}$ Bechelli LM. Prospects of global elimination of leprosy as a public health problem by the year 2000. Int J Lepr, 1994; 62: 284-92.

7 Katoch K, Ramanathan U, Natrjan M, Bagga AK, Bhatia AS, Saxena RK, Ramu G. Relapses in paucibacillary patients after treatment with three short-term regimens containing rifampin. Int J Lepr, 1989; 57: 458-64.

8 Subcommittee on Clinical Trials of the Scientific Working Group on Chemotherapy of leprosy (THELEP) of UNDP/World Bank/WHO special programme on Tropical Diseases. The THELEP controlled clinical drug trials. Int J Lepr, 1987; 55 Suppl: 864-68.

${ }^{9}$ Sreevatsa, Girdhar BK, Desikan KV. Screening of drug resistant strains of Mycobacterium leprae in lepromatous leprosy patients under multidrug treatment. Indian J Med Res, 1988; 87: 139-43.

10 Katoch VM, Katoch K, Ramanathan U, Sharma VD, Shivannavar CT, Datta AK, Bharadwaj VP. Effect of chemotherapy on viability of Mycobacterium leprae as determined by ATP content, morphological index and FDA-EB fluorescent staining. Int J Lepr, 1989; 57: 615-21.

11 Shetty V, Naik, Uplekar M, Antia NH. M. leprae viability in skin and nerve after MDT and their sensitivity to antileprosy drugs. Int J Lepr, 1993; 61 Suppl: CH 14 (A).

12 Ganapati R, Pai VV, Shroff HJ, Gandewar K. Rate of decline in bacterial index in leprosy: observations after three different chemotherapeutic interventions. Int J Lepr, 1997; 65: 264-65.

13 Jamet P, Ji B and the Marchoux Trial Group. Relapse after long term follow-up of multibacillary patients treated by Who multidrug regimen. Int J Lepr, 1995; 63: 195-201.

14 Ponnighaus JM. Diagnosis and management of single lesions in leprosy. Lepr Rev, 1996; 67: 89-94.

15 Single lesion Multicentre Trial Group. Efficiency of single dose multidrug therapy for the treatment of singlelesion paucibacillary leprosy. Indian J Lepr, 1997; 69: 121-30.

16 Katoch K, Natrajan M, Yadav VS, Bhatia AS. response of leprosy patients with single lesions to MDT. Acta Leprol, 1995; 9: 133-37.

17 Pai VV, Revankar CR, Gandewar KL, Bandkar KR, Ganapati R. Clinical assessment of monolesion leprosy casses. Int J Lepr, 1993; 61 Suppl: 30A.

18 Peter Babu, Sudhakar TD, Rajan Babu G. Follow-up of leprosy patients with single patch after multidrug therapy. Indian J Lepr, 1994; 66: 119A. 